\title{
Short cognitive behavioral therapy and cognitive training for adults with ADHD - a randomized controlled pilot study
}

This article was published in the following Dove Press journal:

Neuropsychiatric Disease and Treatment

14 July 2010

Number of times this article has been viewed

\author{
Maarit Virta ${ }^{1,2}$ \\ Anita Salakari' \\ Mervi Antila' \\ Esa Chydenius' \\ Markku Partinen' \\ Markus Kaski' \\ Risto Vataja ${ }^{3}$ \\ Hely Kalska ${ }^{2}$ \\ Matti livanainen' \\ 'Rinnekoti Research Centre, Espoo, \\ Finland; ${ }^{2}$ Department of Psychology, \\ University of Helsinki, Finland; \\ ${ }^{3}$ Kellokoski Hospital, Kellokoski, \\ Finland
}

\begin{abstract}
In clinical practice, a growing need exists for effective non-pharmacological treatments of adult attention-deficit/hyperactivity disorder (ADHD). Here, we present the results of a pilot study of 10 adults with ADHD participating in short-term individual cognitive-behavioral therapy (CBT), 9 adults participating in cognitive training (CT), and 10 controls. Self-report questionnaires, independent evaluations, and computerized neurocognitive testing were collected before and after the treatments to evaluate change. There were distinctive pre-hypotheses regarding the treatments, and therefore the statistical comparisons were conducted in pairs: CBT vs control, CT vs control, and CBT vs CT. In a combined ADHD symptom score based on selfreports, 6 participants in CBT, 2 in CT and 2 controls improved. Using independent evaluations, improvement was found in 7 of the CBT participants, 2 of CT participants and 3 controls. There was no treatment-related improvement in cognitive performance. Thus, in the CBT group, some encouraging improvement was seen, although not as clearly as in previous research with longer interventions. In the $\mathrm{CT}$ group, there was improvement in the trained tasks but no generalization of the improvement to the tasks of the neurocognitive testing, the self-report questionnaires, or the independent evaluations. These preliminary results warrant further studies with more participants and with more elaborate cognitive testing.
\end{abstract}

Keywords: CBT, attention-deficit/hyperactivity disorder, cognitive testing, non-pharmacological treatments

\section{Introduction}

The management of adult attention-deficit/hyperactivity disorder (ADHD) has been under increasing scientific and public debate in recent years, and the need for effective treatments is widely recognized. The most extensively studied treatments are pharmacological (for reviews, see, Dopheide \& Pliszka ${ }^{1}$ and Tcheremissine et $\mathrm{al}^{2}$ ). Pharmacotherapy is thought to help with attention and executive functions deficits, but does not help the individual to develop compensatory strategies. Therefore, in the last 10 years studies on psychological interventions have also emerged. Several group interventions ${ }^{3-9}$ have yielded promising results in treating adults with ADHD. Only a few studies on individual cognitive-behavioral therapy (CBT) have been published. ${ }^{10-12}$ Wilens et al ${ }^{10}$ reported the effectiveness of $10-103$ sessions of individual cognitive therapy, but there was no control group and the study was conducted as a chart review. Safren et a $\mathrm{l}^{12}$ randomized their participants to continued pharmacotherapy alone or a combined pharmacotherapy plus a CBT treatment that they had developed. The combined treatment was found to be associated with better improvement in self-reported symptoms and independent evaluators' ratings as
Correspondence: Maarit Virta Psyyke,Töölönkatu 42, 00250 Helsinki, Finland

Tel +35840 7778281

Fax +358 9445447

Email maarit.virta@psyyke.fi 
compared to pharmacotherapy alone. Rostain and Ramsay ${ }^{11}$ combined pharmacotherapy with 16 sessions in a period of six months of CBT modified to treat adults with ADHD, but they had no control group. They found that the treatment was associated with improvements in the measures used, but as they state, their study design did not allow conclusions about the relative contribution of CBT or pharmacotherapy.

Psychosocial treatments usually focus on compensatory strategies, altering dysfunctional thoughts and attitudes, and improving metacognition, but they do not directly target the core cognitive symptoms of ADHD, such as problems of attention or working memory. In children, some results have been reported on the effectiveness of cognitive training (CT) in treating these deficits. ${ }^{13-16}$ These studies are, however, only case reports or include a small number of patients. There is some evidence that healthy adults may also benefit from computerized working memory training. ${ }^{13,17}$ In adults with ADHD, attention-switching impairment has been shown to ameliorate with short-term computerized training. ${ }^{18}$ To the best of our knowledge, no other studies on CT in adults with ADHD have been reported before.

The aim of this study was to preliminary examine the feasibility and efficacy of short-term individual CBT and CT in adults with ADHD and their impact on ADHD symptoms, mood, quality of life, and cognitive performance. We hypothesized that (i) compared to the controls, the participants in the CBT group would benefit from the treatment, although this may not be as clear as in previously published studies because of the shorter duration of the therapy, and (ii) the participants in the CT group would improve their performance in trained tasks, and this improvement would at least partly generalize to other measures of the same cognitive functions. In addition, we hypothesized that the participants in the CT group would benefit from the treatment as compared to the control group, and this is seen partly in their cognitive performance but not as clearly in their selfreports. Although children with ADHD have been shown to benefit from CT, the adult brain has less plasticity, and thus the treatment gain is hypothesized to be smaller in adults, and (iii) the participants in CBT would benefit more than the participants in CT.

\section{Method}

\section{Participants}

The participants were recruited by announcements placed in an ADHD magazine and an adult ADHD internet discussion forum, and by informing local physicians and clinics specialized in treating ADHD in adults. The inclusion criteria were as follows: (i) 18-49 years of age; (ii) ADHD diagnosis made by a physician; (iii) no diagnosis of psychosis, severe depression or paranoia; (iv) deficits of attention, executive functions or working memory identified in a neuropsychological evaluation that had been made earlier; (v) no current alcohol dependency or drug use; (vi) not receiving a disability pension; (vii) no participation in our previous group rehabilitation study; (viii) currently not undergoing any other psychological rehabilitation; and (ix) no medication or medication that has been stable for at least three months.

In total, 71 interested candidates contacted the researchers and were briefly telephone-screened for the inclusion criteria. Of these, 17 individuals were excluded for not meeting all the inclusion criteria: 11 for having no neuropsychological examination and six for reasons such as diagnosis of psychosis, severe depression or paranoia, older age, retired, or current psychological rehabilitation. If the medication was not stabilized, the candidate was put on a waiting list until this criterion was met.

A total of 54 potential candidates were invited to an interview with a psychologist. The aim of the interview was to screen for the inclusion criteria more closely, to explain the study protocol in more detail and to obtain the informed consent of each candidate before recruitment. Medical records were evaluated to ensure the diagnosis of ADHD and the accurate fulfillment of inclusion criteria 3 and 4. Seven candidates withdrew from the study before the interview or did not attend the interview. At the end of the interview, the candidates filled in a questionnaire of detailed background information and the Wender Utah Rating Scale (WURS). ${ }^{19}$ On the basis of the collected information, the psychologists (AS, MV) verified that the candidate met both the DSM IV criteria for $\mathrm{ADHD}^{20}$ and the criteria for the study. Only one candidate was excluded at this stage because the required neuropsychological deficits were lacking. Thus there were 46 participants who were randomly assigned to one of four groups: hypnotherapy, individual CBT, computerized CT, or the control group. Four of participants accepted for the study withdrew their participation and three quit during the study (two in the CBT and one in the CT group). Thus there were a total of 39 participants. Here we present the results for the CBT, CT and control groups; the results of the hypnotherapy group are published elsewhere. ${ }^{21}$

There were 10 participants in the CBT and control groups, and nine in the CT group. The demographic data of the groups are presented in Table 1. Five of the 10 participants of the CBT group were receiving medication for ADHD, and all of them took methylphenidate. One participant ceased taking 
Table I Characteristics of the participants at the beginning of the treatment

\begin{tabular}{|c|c|c|c|}
\hline & CBT & CT & Control \\
\hline Participants (n) & 10 & 9 & 10 \\
\hline Age: mean (range) & $38.2(25-49)$ & $32.0(2 \mathrm{I}-44)$ & $34.0(22-49)$ \\
\hline Gender: man/woman & $3 / 7$ & $7 / 2$ & $4 / 6$ \\
\hline $\begin{array}{l}\text { Education: } \\
\text { compulsory/additional }^{2}\end{array}$ & $1 / 9$ & $2 / 7$ & $1 / 9$ \\
\hline Work/study: yes/nob & $4 / 6$ & $7 / 2$ & $7 / 3$ \\
\hline ADHD medication $(n)$ & 5 & 5 & 7 \\
\hline $\begin{array}{l}\text { Antidepressive } \\
\text { medication (n) }\end{array}$ & 2 & I & 2 \\
\hline $\begin{array}{l}\text { Any psychiatric } \\
\text { comorbidity (n) }\end{array}$ & 7 & 4 & 3 \\
\hline Depression (n) & 6 & I & 3 \\
\hline Anxiety (n) & 0 & I & 2 \\
\hline Personality disorder (n) & 1 & 3 & 0 \\
\hline Wurs score: mean (SD) & $53.1(12.3)$ & $61.2(11.2)$ & $51.0(15.3)$ \\
\hline $\begin{array}{l}\text { Severity of ADHD (CGI) } \\
\text { Mean (SD) }\end{array}$ & $3.8(0.8)$ & $3.4(0.5)$ & $3.4(0.5)$ \\
\hline
\end{tabular}

Notes: a Compulsory = the participant had completed only lower secondary education (ie, Finnish compulsory education); 'Work/study yes = the participant was working (at least in a half-time job) or studying.

Abbreviations: $\mathrm{CBT}$, cognitive behavioral therapy; $\mathrm{CT}$, cognitive training.

her medication during the rehabilitation, and one added a short-acting methylphenidate Ritalin to the previous longacting methylphenidate Concerta. Five of the nine participants of the CT group were receiving medication for ADHD: four of them took methylphenidate and one modafinil. One participant ceased taking her methylphenidate medication and one changed from Concerta to Equasym, which is a methylphenidate with shorter duration, during the rehabilitation. Seven of the 10 participants of the control group received medication for ADHD: five took methylphenidate, one received modafinil, and one received atomoxetine (which was changed to methylphenidate during the study).

The three groups did not differ, as analyzed by an analysis of variance or Chi-Square test, in age, gender, education, work status, WURS score, severity of ADHD (measured by Clinical Global Impressions, CGI at the baseline) or number of participants having psychiatric comorbidity (all $P \mathrm{~s}>0.05$ ).

None of the participants in the control group received any treatment during the follow-up period. After the follow-up period, eight of them participated in group rehabilitation ${ }^{7}$ and the remaining two in individual rehabilitation.

The study was approved by the Ethics Committee of Helsinki University Central Hospital, Finland and performed in accordance with the ethical standards laid down in the 1964 Declaration of Helsinki. All participants gave their written informed consent prior to participating in the study. Participation was free of charge.

\section{Cognitive-behavioral therapy}

The themes of the treatment sessions were selected to cover the main symptoms set out in the DSM-IV diagnostic criteria $^{20}$ and by Brown. ${ }^{22,23}$

The CBT consisted of 10 weekly sessions led by a psychologist experienced in ADHD and training in CBT (AS). The themes and contents of the sessions are presented in Table 2. The first six sessions and the last session had same content for all the participants, although allowing for some individual modification. Sessions seven, eight and nine were individually tailored. A limit was set at two sessions per theme. For example, it was possible to have self-esteem (session six) as one of the individually chosen sessions or impulsivity (not in sessions one to six) in two individual sessions. The psychologist followed a written manual. ${ }^{24}$ The sessions were semi-structured so as to allow individual treatment. The most important points and tasks at hand were illustrated using printed material and a whiteboard, and at the end of the session, the material discussed was distributed to the participants in written form. In addition, the participants were given homework related to the theme discussed. Each session followed the same procedure: discussion of the previous homework and theme, introduction and discussion

Table 2 Content of the semi-structured cognitive-behavioral therapy (CBT)

\begin{tabular}{l} 
Session I: Goals and symptoms of ADHD \\
Goals and overview of the therapy \\
Symptoms of AD/HD \\
Session 2: Attention \\
Different aspects of attention \\
AD/HD and difficulties in attention and attention-related functions \\
Techniques for relieving attentional problems \\
Session 3: Motivation and initiation of activities \\
Motivation \\
AD/HD and difficulties in getting things started \\
Techniques for improving motivation and initiation \\
Session 4: Organization and planning \\
AD/HD and difficulties in organizing and planning \\
Techniques for organizing time schedules and environment \\
Session 5: Stress management and relaxation \\
Stress and stress management \\
Relaxation exercise \\
Session 6: Self-Esteem \\
Negative beliefs and self-esteem \\
Techniques for improving self-esteem and reformulating negative beliefs \\
Sessions 7 to 9: Individually chosen topics \\
For example, memory techniques, managing impulsivity, anger \\
management or an extra session on some of the previous themes 2-6 \\
Session I0: The continuation of the process, \\
ending the rehabilitation \\
Reviewing of the main points \\
Future challenges and dealing with relapses \\
\hline
\end{tabular}


of the new theme, and assignment of the new homework and distribution of the written material. The duration of a session was approximately 60 minutes.

\section{Cognitive training}

A CT program was developed to include the training of attention, executive functions, and working memory as widely as possible within one hour of computerized, systematic training. The tasks are presented in Table 3. The tasks involved domains of attention as follows: focus-executive (tasks 2 and 7), sustain (1A-D, 4A), encode (5), and shift (6), ${ }^{25}$ and focused (all tasks), sustained (1A-D, 2, 3, 4A-B, 5, 9), selective (1E), alternating (6), and divided (4C, 7, 8) attention. ${ }^{26,27}$ Some of the tasks required executive functions (task 1E: response inhibition; Tasks $4 \mathrm{C}$ and 6: cognitive flexibility) and working memory $(3,5,9)$.

Table 3 Content of the cognitive training (CT)

\section{Task I: Continuous performance tasks (CPT)}

IA-IC: Letters are presented consecutively on the screen for 3 minutes. In IA, the participant has to press a button when the target letter appears. In IB, he/she has to press a button when either of the two target letters appears. In IC, the participant has to press a button when the target letter appears after a predetermined cue letter. ID: Letters are presented via loud speaker for 3 minutes, and the participant has to press a button each time the target letter is heard. IE: Letters are presented on the screen and via loud speaker for 3 minutes. The participant has to press a button when the target letter appears on the screen and simultaneously ignore distracting letters he/she hears from the loud speaker.

Adjustment of the difficulty level: When a participant succeeds in performing at a predetermined level, the time interval between the onsets of the stimuli is shortened.

\section{Task 2: Digit search}

A $20 \times 20$ matrix of single digits is presented on the screen. The participant has to search for all the target digits as quickly as possible and click on them with a mouse. There are three different kinds of tasks: a search for two targets, three targets or three targets with an extra rule (a predetermined cue digit has to precede the third target digit).

Adjustment of the difficulty level: When a participant succeeds at the simplest task at a predetermined level, a more difficult task is then presented.

Task 3: Circle-letter sequences

Circles are presented on the screen. They start to turn red in random order, and a letter appears shortly under each red circle. After the presentation of the whole sequence, the participant is asked to remember which letter appeared below which circle in random order. Each sequence length is presented three times, and if a participant gets two or three of them correct, a longer sequence is presented.

Adjustment of the difficulty level: Circle-letter sequences are lengthened according to the participant's performance.

\section{Task 4: CPT and a simultaneous task}

4A: Colored geometric figures are presented on the screen one at a time for 3 minutes. The participant has to press a button when the target figure (of the correct shape and color) appears. 4B: Simple addition problems are presented on the screen for 3 minutes. The participant has to press a button when the calculation is incorrect. 4C: The participant performs tasks 4A and 4B simultaneously for 3 minutes.

Adjustment of the difficulty level: When a participant succeeds in performing at a predetermined level, the time interval between the onsets of the stimuli is shortened.

\section{Task 5: Digit arrangement}

Simple digits are presented in random order via loud speaker. The participant has to arrange them in the right order (eg, I-5-0-I-7 is 0-I-I-5-7). Each sequence length is presented twice, and if a participant gets either one of them correct, a longer sequence is presented.

Adjustment of the difficulty level: Digit sequences are lengthened according to the participant's performance.

\section{Task 6: Alternating rules}

Groups of alternating colored letters are presented on the screen together with alternating rules for the task (eg, press a button when there is a green A/at least three C's/not a blue K). The participant presses the button according to the rules for 3 minutes.

Adjustment of the difficulty level: Stimuli are presented more quickly when participant succeeds in performing at a predetermined level. At the easiest level, a beep sound indicates when a rule is changed.

Task 7: Digit-letter search

A $20 \times 20$ matrix of single digits is presented on the screen. The participant has to search for the target digits as quickly as possible and click on them with a mouse. Simultaneously, he/she hears letters and has to press a button each time the target letter is heard.

Adjustment of the difficulty level: When a participant succeeds in performing at a predetermined level, the time interval between the onsets of the stimuli is shortened.

\section{Task 8: Counting while reading}

A text is presented on the screen. The participant reads it aloud and simultaneously counts silently (mentally) how many target letters are imbedded in the text.

Adjustment of the difficulty level: The text is lengthened when the participant succeeds in performing at a predetermined level. At the easiest level, the target letters are presented in bold.

\section{Task 9: Circle sequences}

9A: A $5 \times 5$ matrix of circles is presented on the screen. Some of the circles turn red in random order. After the presentation, the participant has to click on the circles with a mouse in the same order. 9B: The task is the same as 9A except that the circles are to be clicked in reverse order. Adjustment of the difficulty level: The circle sequence is lengthened according to the participant's performance. 
The CT consisted of 20 sessions taking place twice a week and led by a psychologist (AS). Tasks 1A, B and C were practiced only in every third session. All the other tasks were completed in every session. Each participant received feedback on the screen immediately after each task, and the feedback was discussed with the psychologist. There were several difficulty levels in the tasks - at least three in each, and in most of the tasks at least six - and the tasks were adjusted to a suitable level on a trial-by-trial basis (Table 3 ). The duration of a session was approximately 60 minutes.

Because of the large number of tasks and variables, the participants' performance change is only presented for four tasks in the Results section, namely for tasks 3, 5, 4C and 7. In tasks 3 and 5, the length of the longest correctly remembered/arranged sequence in every session is taken as the result. In tasks 4 and 7, the difficulty level used at each session is the result. In task 7, there were seven difficulty levels in which the visual stimuli were always similar, but the presentation rate of the auditory stimuli varied - that is, the time elapsed from the onset of the previous letter to the onset of the next letter was either 2200, 1900, 1600, 1300, 1100,900 or $850 \mathrm{~ms}$. At the first session, all the participants started at level three $(1600 \mathrm{~ms})$. If the participant's responses were more than $90 \%$ correct and he or she made less than five erroneous responses in both tasks, level four (1300 ms) was presented at the next session. If the participant got less than $70 \%$ correct or made more than 10 errors, the easier level two (1900 ms) was presented at the next session. In task 4 , the same rules were applied, and the time elapsed from the onset of the previous item to the onset of the next item was $4000,3500,3000,2500,2000,1500,1200,1100$, and $1000 \mathrm{~ms}$ (9 levels), starting at level three (3000ms).

\section{Outcome measures}

As outcome measures we used self-report questionnaires, independent evaluations, and computerized neurocognitive testing. For the treatment groups, data were collected before the treatment (T1) and after the treatment (T2). Data obtained from the control group were also collected on two occasions. T2 measurements were scheduled to be taken about 11 or 12 weeks after T1 to correspond to the time in the treatment group. The mean time elapsed between $\mathrm{T} 1$ and T2 (the questionnaires and the neurocognitive testing) was 82 days (range 63-112) for the CBT group, 94 days (range 56-115) for the CT group, and 81 days (range 69-91) for the control group. The T1 questionnaires and testing were completed 0-12 days before the first CBT or CT sessions, and the T2 measures 0-9 days after the last session. Independent evaluations were collected before T1 and after T2, within two weeks of the collection of self-report questionnaires and neurocognitive testing (with one exception of three weeks). The independent evaluator was a clinical psychologist (MA) who was blind to the actual study group of participants. The measures used were:

\section{Brown Attention Deficit Disorder Scale - Adult Version (BADDS) ${ }^{28}$}

The BADDS is a 40 -item inventory of which we used the self-report version. From BADDS, a total score and scores of the five subdomains of activation, attention, effort, affect, and memory were derived. Higher scores indicate a greater impairment.

\section{World Health Organization's Adult ADHD Self-report Scale (ASRS) ${ }^{29}$}

The ASRS is an 18-item scale reflecting the DSM-IV criteria modified for adults. We used both self-report and independent evaluator measurements. We report the total score in which the higher the scores, the greater the impairment.

\section{Symptom Check List (SCL-90) $)^{30}$}

The SCL-90 is a 90-item self-report scale for the measurement of psychiatric symptoms. Several subscales can be calculated, but we used the total score. Moreover, a 16-item sum score (SCL-16) reflecting the characteristics prominent in $\mathrm{ADHD}^{3}$ was calculated from the SCL-90. The higher the scores, the greater were the symptoms.

\section{Beck Depression Inventory, second edition (BDI-II) ${ }^{31}$}

The BDI II is a 21-item scale that evaluates current selfreported symptoms of depression. Higher scores reflect greater problems.

\section{Quality of Life Enjoyment and Satisfaction Questionnaire (Q-LES-Q) ${ }^{32}$}

The Q-LES-Q is a 93-item self-report scale, from which 91 items can be grouped into eight subscales that indicate satisfaction with physical health, subjective feelings, work, household duties, school, leisure activities, social relationships, and general activities. We combined the work and school subscales into a work/study subscale. When a participant gave both scores, the main score was used (ie, if the participant was working full time and also taking some educational courses, then the work score was used). Higher scores indicate greater enjoyment or satisfaction. The scores are reported as a percentage of the maximum score. 


\section{Clinical Global Impressions (CGI) ${ }^{33}$}

CGI was completed by the independent evaluator. At T1, the severity of ADHD was evaluated according to CGI, which is a single 7-point rating scale of functioning varying from $1=$ normal, not at all ill, to $7=$ among the most extremely ill patients. At T2, global improvement was assessed using a 7-point scale varying from $1=$ very much improved, to $7=$ very much worse $(4=$ no change $)$. Classes 1 to 3 were combined to represent improvement, and classes 4 to 7 were combined to represent non-improvement.

\section{CNS Vital Signs (CNSVS) $)^{34,35}$}

CNSVS is a computerized neurocognitive test battery comprising seven neuropsychological tests: verbal memory, visual memory, finger tapping, symbol-digit coding, the Stroop test, the shifting attention test, and the continuous performance test. Five standardized domains were obtained from the tests: memory, psychomotor speed, reaction time, cognitive flexibility, and complex attention. In addition, we used the neurocognition index, which is the mean of the domains. A detailed description of the tests of CNSVS has been given by Gualtieri and Johnson. ${ }^{35,36}$

In addition, the CBT and CT participants evaluated the benefit of the rehabilitation at $\mathrm{T} 2$ using a five-option rating scale of no benefit, minor benefit, moderate benefit, clear benefit, and substantial benefit.

\section{Statistical analyses}

The individual missing values on the questionnaires were substituted with the respondent's mean score. However, no replacements were made in the Q-LES-Q as the scores were calculated as a percentage of the maximum score.

The distribution properties of the variables were inspected visually and with Shapiro-Wilk tests. Parametric tests were chosen for the statistical analyses. Since we had clear prehypotheses to be tested, comparisons were made for three different pairings: the CBT group vs the control group, the CT group vs the control group, and the CBT group vs the CT group. A two-way mixed ANOVA with one between factor, group, and one within factor, time (T1 vs T2) was performed. The effect sizes were quantified by partial eta squared $\eta_{p}^{2}$. When the ANOVA was significant or almost significant $(P<0.10)$ and the effect size large $\left(\eta_{p}^{2}>0.138\right)$, paired $\mathrm{t}$-tests were also performed for all groups separately. Changes in CGI were analyzed using the Chi-square test $\left(\chi^{2}\right)$.

\section{Results}

The mean scores of the self-report and computerized neurocognitive test battery measures for the CBT, CT and control groups are presented in Table 4. The treatment lasted for 10.5 weeks (range 9-15 weeks) for the CBT and 12.2 weeks (range 7.6-15.3 weeks) for the CT.

\section{Cognitive behavioral therapy group versus controls}

To compare the CBT group with the control group, a twoway ANOVA with one between-factor, group (CBT vs control), and one within-factor, time (T1 vs T2), was performed. There was a significant Time $\times$ Group interaction in BADDS attention $\left[F(1,18)=7.24, P<0.05, \eta_{p}^{2}=0.29\right]$, memory $\left[F(1,18)=6.32, P<0.05, \eta_{p}^{2}=0.26\right]$ and total scores $\left[F(1,18)=6.32, P<0.05, \eta_{p}^{2}=0.26\right]$. Moreover, an almost significant interaction was found for the Q-LES-Q work/study subscale $\left[F(1,11)=4.28, P=0.06, \eta_{p}^{2}=0.28\right]$. As seen in Table 4, a decrease in symptoms takes place mainly in participants of the CBT group. No other statistically significant interactions were found in the self-report questionnaires (all $P \mathrm{~s}>0.10$ ). There were also no statistically significant interactions in the standardized domains and neurocognition index for CNSVS (all $P \mathrm{~s}>0.10$ ).

In the control group, there was no difference between $\mathrm{T} 1$ and T2 in any measure (all $P \mathrm{~s}>0.05$ ) in paired t-tests. In contrast, there was a significant decrease of symptoms between $\mathrm{T} 1$ and $\mathrm{T} 2$ in the CBT group in BADDS attention $[t(9)=4.35$, $\left.P<0.01, \eta_{p}^{2}=0.68\right]$, memory $\left[t(9)=2.78, P<0.05, \eta_{p}^{2}=0.46\right]$ and total scores $\left[t(9)=3.96, P<0.01, \eta_{p}^{2}=0.64\right]$.

The participants were classified into two groups according to their individual improvement or nonimprovement during the study. A participant was defined as "improved" when he or she had reduced self-reported symptoms in all ratings of ADHD symptoms, namely the BADDS total score, the SCL-16, and the ASRS. In cases of either symptom elevation or no change in any of the measures, the participant was classified as "not improved". Six of the 10 participants $(60 \%)$ in the CBT group were improved compared with two of the ten $(20 \%)$ in the control group. The difference was almost statistically significant $\left(\chi^{2}=3.33, \mathrm{df}=1, P=0.07\right)$.

According to the independent evaluators' CGI ratings, seven of the $10(70 \%)$ participants in the CBT group and three of the 10 individuals $(30 \%)$ in the control group improved from $\mathrm{T} 1$ to $\mathrm{T} 2$. The difference was almost statistically significant $\left(\chi^{2}=3.20, \mathrm{df}=1, P=0.07\right)$. For the independent evaluator-rated ASRS, no statistically significant interaction was found $\left[F(1,18)=1.67, P=\mathrm{ns}, \eta_{p}^{2}=0.09\right]$.

In the participants' self-evaluations of the treatment benefit, nine of the ten CBT participants reported at least a clear benefit. 
Table 4 Mean (standard deviation) scores for the participants' self-ratings and computerized neurocognitive test battery at TI (before treatment) and T2 (after treatment)

\begin{tabular}{|c|c|c|c|c|c|c|}
\hline & \multicolumn{2}{|l|}{ CBT } & \multicolumn{2}{|l|}{$\mathbf{C T}^{\mathrm{a}}$} & \multicolumn{2}{|l|}{ Controls } \\
\hline & TI & T2 & TI & T2 & TI & T2 \\
\hline \multicolumn{7}{|l|}{ BADDS } \\
\hline Activation & $20.2(2.0)$ & $17.5(4.5)$ & $17.6(3.7)$ & $15.0(4.8)$ & $17.2(6.1)$ & $16.8(6.1)$ \\
\hline Attention & $22.3(3.3)$ & $18.3(5.0)$ & $17.8(3.1)$ & $16.2(5.2)$ & $19.3(3.3)$ & I9.1 (3.2) \\
\hline Effort & I $8.4(4.3)$ & $15.6(4.4)$ & $16.8(4.9)$ & $13.9(6.2)$ & $15.6(5.9)$ & I4.4 (6.I) \\
\hline Affect & $11.6(2.2)$ & $9.6(2.8)$ & $10.0(3.1)$ & $6.9(4.2)$ & $9.1(3.1)$ & $8.7(4.4)$ \\
\hline Memory & $12.3(4.5)$ & $10.5(5.0)$ & $9.7(4.6)$ & $9.2(3.0)$ & $10.4(4.4)$ & $11.0(4.1)$ \\
\hline Total & $84.8(12.8)$ & $71.5(18.6)$ & $71.8(13.5)$ & $61.2(17.8)$ & $71.6(16.3)$ & $70.0(14.2)$ \\
\hline $\mathrm{BDI} \|^{\mathrm{a}}$ & $13.6(5.6)$ & $9.0(8.3)$ & $6.4(5.5)$ & $4.8(5.8)$ & $11.0(7.7)$ & $9.5(I 1.6)$ \\
\hline SCL-90 & $95.4(2 \mid .2)$ & $81.9(27.7)$ & $67.6(31.4)$ & $68.0(34.0)$ & $94.1(37.8)$ & $89.7(48.0)$ \\
\hline SCL-16 & $31.0(7.4)$ & $25.2(5.3)$ & $20.9(8.1)$ & $22.9(9.2)$ & 31.5 (1I.9) & 30.2 (11.5) \\
\hline ASRS & $50.7(10.7)$ & $45.4(11.7)$ & $45.7(6.7)$ & $4 I .2(4.9)$ & $50.8(7.7)$ & $47.8(12.0)$ \\
\hline \multicolumn{7}{|l|}{ Q-LES-Qc } \\
\hline General & $55.7(8.6)$ & $60.9(14.5)$ & $60.3(12.6)$ & $65.2(14.4)$ & $54.4(I I .7)$ & $59.2(21.0)$ \\
\hline Work/study ${ }^{\mathrm{b}}$ & $58.7(23.9)$ & $72.7(15.2)$ & $66.1(15.8)$ & $67.5(19.0)$ & $73.3(8.2)$ & $59.0(25.6)$ \\
\hline \multicolumn{7}{|l|}{ CNSVS } \\
\hline Neurocognitive index & $85.4(15.1)$ & $97.6(9.2)$ & $94.3(9.5)$ & $97.6(11.9)$ & $97.1(13.0)$ & $102.5(10.1)$ \\
\hline Memory & $92.9(19.8)$ & $102.1(12.8)$ & $89.0(16.3)$ & $91.4(2 \mid .2)$ & $104.7(15.4)$ & $111.6(12.4)$ \\
\hline Psychomotor speed & $80.3(15.9)$ & $81.1(13.8)$ & $94.0(16.3)$ & $98.8(6.6)$ & $90.2(14.2)$ & $90.8(16.0)$ \\
\hline Reaction time & $82.9(24.3)$ & $94.9(14.0)$ & $90.4(12.5)$ & $94.6(14.8)$ & $96.2(10.7)$ & $99.3(12.3)$ \\
\hline Flexibility & $82.9(21.5)$ & $104.9(15.5)$ & $98.7(14.3)$ & I04.I (I7.I) & $96.1(24.7)$ & $106.3(18.4)$ \\
\hline Attention & $88.0(19.3)$ & $105.9(8.9)$ & $98.7(16.6)$ & $98.1(15.8)$ & $98.0(17.6)$ & I04.4 (16.9) \\
\hline
\end{tabular}

Notes: $\mathrm{N}=10$ except; ${ }^{\mathrm{N}} \mathrm{N}=9$ in the cognitive training (CT) group; ${ }^{\mathrm{b}} \mathrm{A}$ participant was included only if scores were available for both $\mathrm{TI}$ and $\mathrm{T} 2$. In the cognitive behavioral therapy (CBT) group $N=7$, in the $C T$ group $N=8$ and in the control group $N=6$; 'All subscales of the $Q-L E S-Q$ were analysed, but no statistically significant results were found. Only the results of the general and combined work/study subscale are presented here.

\section{Cognitive training group versus controls}

The participants' performance in the trained tasks was analyzed to find out whether there was task-specific improvement related to the rehabilitation. In task 3 , the participants were able to remember correctly a sequence of 3.22 (SD 1.48) items in the first session and 5.44 (1.94) in the last session. This difference is statistically significant $\left[t(8)=4.26, P<0.01, \eta_{p}^{2}=0.66\right]$. In task 5 , the participants were able to arrange correctly a sequence of 6.56 (SD 1.01) items in the first session and 8.33 (3.40) in the last session, reaching statistical significance $[t(8)=2.16$, $\left.P<0.05, \eta_{p}^{2}=0.44\right]$. The rising learning curve of participants' performance in tasks $4 \mathrm{C}$ and 7 is presented in Figure 1.

In the self-report questionnaires, when analyzing the $\mathrm{CT}$ and control groups with an ANOVA, there was an almost significant Time $\times$ Group interaction between $\mathrm{T} 1$ and $\mathrm{T} 2$ in BADDS affect $\left[F(1,17)=3.23, P=0.09, \eta_{p}^{2}=0.16\right]$. In a paired t-test, the $\mathrm{CT}$ group had a significant decrease in symptoms between T1 and T2 $\left[t(8)=3.18, P<0.05, \eta_{p}^{2}=0.56\right]$ in BADDS affect (see Table 4). There were no statistically significant interactions in other self-report questionnaires (all $P \mathrm{~s}>0.10$ ). No statistically significant interactions were found in measures of CNSVS (all $P_{\mathrm{S}}>0.10$ ).

Two of the nine participants (22\%) in the CT group were classified as improved compared with two of the ten (20\%) in the control group. Thus, the groups did not $\operatorname{differ}\left(\chi^{2}=0.01\right.$, $\mathrm{df}=1, P=\mathrm{ns})$.

According to the independent evaluators' CGI ratings, two of the nine (22\%) participants in the CT group and three of the 10 individuals $(30 \%)$ in the control group improved

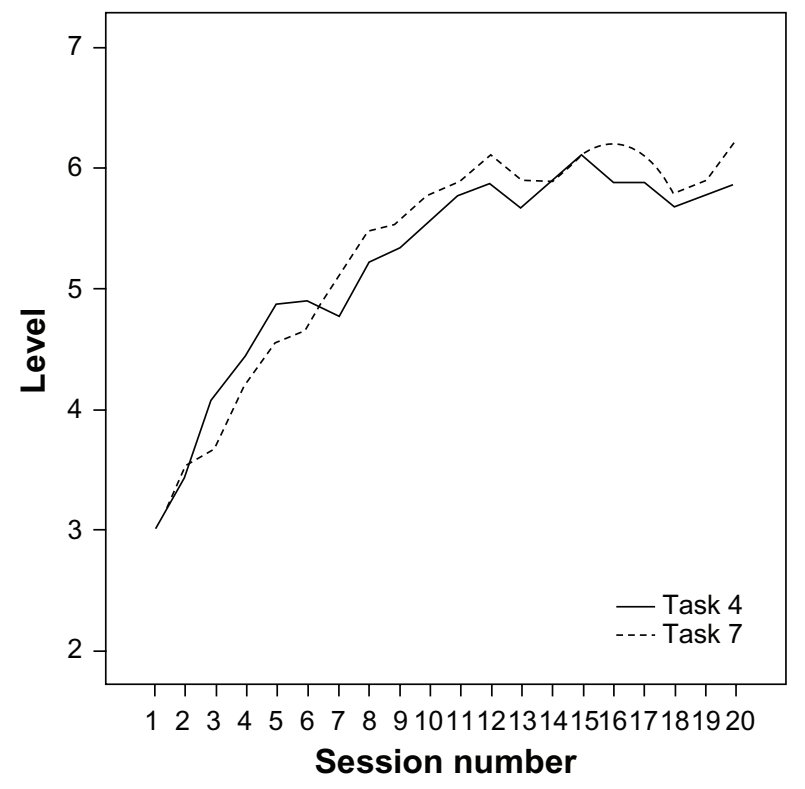

Figure I Level of performance for tasks $4 \mathrm{C}$ and 7 during the rehabilitation in every session. 
from $\mathrm{T} 1$ to $\mathrm{T} 2$. This difference was not statistically significant ( $\left.\chi^{2}=0.15, \mathrm{df}=1, P=\mathrm{ns}\right)$. Moreover, there were no statistically significant interactions for the independent evaluatorrated ASRS $\left[F(1,18)=0.55, P=\mathrm{ns}, \eta_{p}^{2}=0.03\right]$.

In the participants' self-evaluations of the treatment benefit, four of the nine CT participants reported at least a clear benefit.

\section{Cognitive-behavioral therapy versus cognitive training}

There was an almost significant Time $\times$ Group interaction of symptoms between $\mathrm{T} 1$ and $\mathrm{T} 2$ in BADDS attention $\left[F(1,17)=3.12, P=0.09, \eta_{p}^{2}=0.15\right]$ and the SCL-16 $\left[F(1,17)=4.20, P=0.06, \eta_{p}^{2}=0.20\right]$, suggesting more improvement in the CBT group (see Table 4). No other statistically significant interactions were found in the self-report measures. Thus, the amelioration of symptoms in the two groups did not statistically differ. No statistically significant interactions were found for CNSVS either (all $P_{\mathrm{S}}>0.10$ ).

As previously mentioned, six of the 10 participants $(60 \%)$ in the CBT group were classified as improved compared with two of the nine $(20 \%)$ in the CT group. This difference was almost statistically significant $\left(\chi^{2}=2.77, \mathrm{df}=1, P=0.10\right)$.

According to the independent evaluators' CGI ratings, seven of the $10(70 \%)$ participants in the CBT group and two of the nine individuals $(22 \%)$ in the CT group improved from $\mathrm{T} 1$ to $\mathrm{T} 2$. This difference was statistically significant $\left(\chi^{2}=4.34\right.$, df $\left.=1, P<0.05\right)$. No statistically significant interaction for the independent evaluator-rated ASRS was found $\left[F(1,17)=2.52, P=\mathrm{ns}, \eta_{p}^{2}=0.13\right]$.

\section{Discussion}

The aim of the present pilot study was to examine the potential feasibility and efficacy of short-term CBT and CT in treating adults with ADHD. The influence of the treatments on ADHD symptoms, mood, quality of life, and cognitive performance were evaluated. Both treatments were found to be quite acceptable and tolerable to the participants: only two participants in the CBT group and one in the CT group quit during the rehabilitation. Since there were three distinctive pre-hypotheses, the CBT group was compared to the control group, the CT group to the control group, and the CBT group to the CT group.

\section{Short-term cognitive-behavioral therapy}

The self-reported symptoms of participants in the CBT group decreased significantly (or almost significantly with large effect sizes) in BADDS attention, memory, and total scores, and on the Q-LES-Q work/study subscale, as compared to the control group. When individual percentages of change were investigated, six of the 10 participants in the CBT group had improved compared with two of the 10 in the control group. According to the independent evaluator's CGI ratings, seven of the 10 participants in the CBT group and three of the 10 in the control group, improved from T1 to T2. There were no statistically significant interactions in the standardized domains and neurocognition index of CNSVS. The participants' self-evaluations regarding the usefulness of the program were quite high; nine of the $10 \mathrm{CBT}$ participants reported at least a clear benefit.

The improvement found in self-report questionnaires, self-evaluations, and independent evaluator's ratings is consistent with our pre-hypothesis and previous studies of individual CBT in adult ADHD. ${ }^{10-12}$ It is, nevertheless, somewhat difficult to compare the present and previous studies since they differ in inclusion criteria, study design, and measures used. For example, our participants seemed to benefit less than the participants in Rostain and Ramsay's ${ }^{11}$ study, where the BADDS total scores decreased from 70.5 to 49.9 (ours decreased from 84.8 to 71.5 ). However, their participants received a combined treatment (CBT and medication), whereas the participants of our study were either non-medicated or stably medicated before the treatment. The treatment duration in Rostain and Ramsay's study was 16 sessions over 6 months as compared to the 10 sessions and 2.5 months of the present study. Since ADHD is a developmental disorder with long-lasting and often pervasive problems, it is reasonable that CBT of longer duration may be needed to develop and establish adaptive coping skills. However, some encouraging improvement was seen with our short-term CBT, although not as clearly as in previous studies with longer interventions. We hypothesize that this kind of short intervention may be useful right after a diagnosis or for milder cases, and longer-term treatments could be used for those with more pervasive problems.

\section{Cognitive training}

A training benefit was clearly seen in the trained tasks, where most of the participants improved their performance markedly. However, the benefit was not seen in CNSVS or in most of the self-report questionnaires. Only a decrease in the BADDS affect score was observed (almost statistically significant, large effect size). In addition, no improvement was found in individual percentages of change or independent evaluations.

The improvement in trained tasks is in line with our pre-hypothesis. However, it was also hypothesized that this 
task-specific improvement would generalize at least in some extent to cognitive measures and perhaps not as strongly to the self-report questionnaires. Against our expectations, this was not found. In a previous study of working memory training with nonmedicated ADHD children, learning was found to generalize to nontrained working memory tasks, other nontrained measures of executive function, and parent ratings, but not to teacher ratings. ${ }^{14}$ The same study group has also found that working memory training improves performance in healthy adults and that this improvement also transfers to other measures. ${ }^{13,17}$ Also short-term attention-switching training has been shown to transfer to new tasks of attention-switching in adults with ADHD. ${ }^{18}$ Thus, our findings also disagree with the previous studies. However, this may not necessarily mean that adults with ADHD do not benefit from this type of CT. It is possible that CNSVS, which was used as the cognitive measure was too easy for the participants (see the general discussion section), or that the CNSVS tasks did not measure the same components of attention and executive functions that were trained. The intensity of training might also be influential. In addition, the improvement seen in BADDS affect is intriguing. This may be just a random result due to the small sample size, or it may reflect the training of impulse control.

To the best of our knowledge, this is the first study examining the effects of CT in adults with ADHD. Clearly, the possibilities of $\mathrm{CT}$ for adult ADHD need to be studied more in the future.

\section{Comparison of the two treatments}

The two treatments studied here were equal in length, but differed in intensity: CBT took place once a week, whereas CT took place twice a week. The self-reported symptoms of CBT participants decreased almost significantly more (with a large effect size) in BADDS attention and the SCL-16 as compared to the CT participants. No statistically significant differences were found in CNSVS. According to the individual percentages of change (six of the 10 in the CBT group and two of the nine in the CT group were classified as improved) and the independent evaluators' CGI ratings (seven of the 10 in the CBT group and two of the nine in the CT group improved), the participants in the CBT group benefited more.

Thus, at the general level, even our very short-term CBT seemed to be more effective than CT with more sessions. This is in line with our pre-hypothesis. However, at the level of individual measures, the evidence is limited. Rostain and Ramsay have argued that pharmacotherapy is a bottom-up treatment targeting the core symptoms of ADHD, and that CBT represents a top-down approach. ${ }^{11}$ We assume that CT is mainly a bottom-up treatment, but since the participants received feedback from the tasks, and some of them were active in developing and testing their own strategies, a topdown approach was also embedded in it. However, it seems that CT is not as beneficial as the top-down CBT.

\section{General discussion}

More than half of our participants were medicated at the beginning of the treatment (five of the 10 in the CBT group, five of the nine in the CT group, and seven of the 10 in the control group). Their medication was required to be stabilized for three months before the treatment, and there were only minor changes in medication during the treatment. It can be assumed that the positive effects of pharmacotherapy were already obtained before entering the study, and therefore, the treatment benefits were not related to medication. Safren et $\mathrm{al}^{12}$ found that better treatment results are gained when adding psychosocial treatment to pharmacotherapy. This is in line with our results where CBT seemed to be effective for already medicated participants.

Previously, only two psychosocial intervention studies of adult ADHD have used cognitive functioning as an outcome measure. ${ }^{3,8}$ In a study on mindfulness training, ${ }^{8}$ improvement in tasks of attention and cognitive inhibition was found. In a pilot study using a structured skills training program in a group setting, ${ }^{3}$ neuropsychological testing was used at baseline and following treatment, and some tendency towards improvement was found. However, a practice effect cannot be ruled out in either study since neither had a control group, ${ }^{8}$ or when present, the control group did not undergo neuropsychological testing. ${ }^{3}$ In our study, no treatment-related improvement was found in cognitive functioning as measured by CNSVS, a computerized neurocognitive test battery; that is, no more improvement was seen in the treatment groups than in the control group. In a study of Gualtieri and Johnson ${ }^{34}$ employing CNSVS, adults with ADHD were found to be impaired in measures of psychomotor speed, reaction time, cognitive flexibility, and attention when compared to normal controls. In our sample, some participants had difficulties in cognitive functioning measured by CNSVS, whereas some had no difficulties even though deficits had been reported in their previous neuropsychological examinations. The ceiling effect was also present in many participants in some of the tasks, especially in CPT ( 25 of the 29 participants received the maximum score of correct responses at T1) and in the Stroop test (26 of the 29 participants). Therefore we suggest that other, perhaps more sensitive, measures of cognitive functioning may also be needed. 
Although it is not possible with this small sample to predict which participants would have the most improvement from CBT, we agree with Rostain and Ramsay ${ }^{11}$ that it would probably be those with a "more reasonable assessment of the treatment process and whose expectations about outcome were more realistic". Our clinical impression was that the participants who were most motivated to learn new adaptive skills benefited most from the CBT. Also, those who benefited from the $\mathrm{CT}$ used their training sessions as an opportunity to develop and test their own ideas and strategies and did not just "mechanically" complete the tasks.

\section{Concluding remarks}

There are some limitations of the study that should be considered when interpreting the results. First, the sample size was small in every group: 10 participants in the CBT group, nine participants in the CT group, and 10 in the control group. Thus, the results must be considered with caution. Second, although the participants were randomly assigned to either control or active treatment groups, the control group had no intervention during the follow-up. Therefore, possible placebo effects cannot be ruled out. Even so, blinded or sham treatments would be almost impossible to carry out reliably when studying these kinds of psychosocial treatments. The third limitation is related to the severity of the ADHD symptoms of the participants. In the independent evaluator's CGI ratings, the participants were rated from mildly to markedly ill, thus no extreme cases were included. According to CGI, our participants had milder ADHD than, for example, Rostain and Ramsay had in their study, even though our CBT participants' self-ratings in the BADDS total score were higher. ${ }^{11}$ Thus, the independent evaluator's personal rating style may have influenced the results. Also, the recruitment of the participants may have caused some bias towards more motivated and less severely disabled adults with ADHD participating in the study.

Despite these limitations, our study has many strengths. The diagnoses were made by a specialist and duly verified, the outcome measures were wide-ranging (self-report questionnaires, independent evaluations and neurocognitive testing), and we also used a control group and randomization in the study design. Some encouraging treatment benefits were obtained in the group that received short-term CBT, even though most of the participants were already receiving medication. However, CBT and CT studies with a larger sample size and more extensive cognitive testing are needed in the future. Also, the influence of treatment length and intensity needs to be studied.

\section{Acknowledgments}

This study was supported by RAY, Finland's Slot Machine Association. Maarit Virta received funding for preparation of this manuscript from the Rinnekoti Research Foundation. We are grateful to Pekka Lahti-Nuuttila for statistical support. The author report no conflicts of interest in this work.

\section{References}

1. Dopheide JA, Pliszka SR. Attention-deficit-hyperactivity disorder: an update. Psychopharmacol Bull. 2009;29(6):656-679.

2. Tcheremissine OV, Salazar JO. Pharmacotherapy of adult attention deficit/hyperactivity disorder: review of evidence-based practices and future directions. Expert Opin Pharmacother. 2008;9(8):1299-1310.

3. Hesslinger B, Tebartz van Elst L, Nyberg E, et al. Psychotherapy of attention deficit hyperactivity disorder in adults - A pilot study using a structured skills training program. Eur Arch Psychiatry Clin Neurosci. 2002;252(4):177-184.

4. Philipsen A, Richter H, Peters J, et al. Structured group psychotherapy in adults with attention deficit hyperactivity disorder: results of an open multicentre study. J Nerv Ment Dis. 2007;195(12):1013-1019.

5. Solanto MV, Marks DJ, Mitchell KJ, Wasserstein J, Kofman MD. Development of a new psychosocial treatment for adult ADHD. JAtten Disord. 2008;11(6):728-736.

6. Stevenson CS, Whitmont S, Bornholt L, Livesey D, Stevenson RJ. A cognitive remediation programme for adults with attention deficit hyperactivity disorder. Aust N Z J Psychiatry. 2002;36(5):610-616.

7. Virta M, Vedenpää A, Grönroos N, et al. Adults with ADHD benefit from cognitive-behaviorally oriented group rehabilitation - A study of 29 participants. J Atten Disord. 2008;12(3):218-226.

8. Zylowska L, Ackerman DL, Yang MH, et al. Mindfulness meditation training in adults and adolescents with ADHD: a feasibility study. $J$ Atten Disord. 2008;11(6):737-746.

9. Salakari A, Virta M, Grönroos N, et al. Cognitive-behaviorally-oriented group rehabilitation of adults with ADHD: results of a 6-month follow-up. J Atten Disord. 2010;13(5):516-523.

10. Wilens TE, McDermott SP, Biederman J, Abrantes A, Hahesy A, Spencer TJ. Cognitive therapy in the treatment of adults with ADHD: A systematic chart review of 26 cases. J Cogn Psychother. 1999;13(3): 215-226.

11. Rostain AL, Ramsay JR. A combined treatment approach for adults with ADHD - Results of an open study of 43 patients. J Atten Disord. 2006;10(2):150-159.

12. Safren SA, Otto MW, Sprich S, Winett CL, Wilens TE, Biederman J. Cognitive-behavioral therapy for ADHD in medication-treated adults with continued symptoms. Behav Res Ther. 2005;43(7):831-842.

13. Klingberg T, Forssberg H, Westerberg H. Training of working memory in children with ADHD. J Clin Exp Neuropsychol. 2002;24(6): 781-791.

14. Klingberg T, Fernell E, Olesen P, et al. Computerized training of working memory in children with ADHD-a randomized, controlled trial. J Am Acad Child Adolesc Psychiatry. 2005;44(2):177-186.

15. Shalev L, Tsal Y, Mevorach C. Computerized progressive attentional training (CPAT) program: effective direct intervention for children with ADHD. Child Neuropsychol. 2007;13(4):382-388.

16. Slate SE, Meyer TL, Burns WJ, Montgomery DD. Computerized cognitive training for severely emotionally disturbed children with ADHD. Behav Modif. 1998;22(3):415-437.

17. Olesen PJ, Westerberg H, Klingberg T. Increased prefrontal and parietal activity after training of working memory. Nat Neurosci. 2004;7(1):75-79.

18. White HA, Shah P. Training attention-switching ability in adults with ADHD. JAtten Disord. 2006;10(1):44-53. 
19. Ward MF, Wender PH, Reimherr FW. The Wender Utah Rating Scale: an aid in the retrospective diagnosis of childhood attention deficit hyperactivity disorder. Am J Psychiatry. 1993;150(6):885-890.

20. American Psychiatric Association. Diagnostic and Statistical Manual of Mental Disorders. 4th ed. Washington, DC: American Psychiatric Association; 1994.

21. Virta M, Salakari A, Grönroos N, et al. Hypnotherapy for adults with attention deficit hyperactivity disorder - a randomized controlled study. Contemp Hypnosis. 2010; In press.

22. Brown TE editor. Attention-Deficit Disorders and Comorbidities in Children, Adolescents, and Adults. Washington DC; American Psychiatric Press; 2000.

23. Brown TE. Attention Deficit Disorder: The Unfocused Mind in Children and Adults. New Haven, CT: Yale University Press; 2005.

24. Virta M, Salakari A, Vataja R, et al. AD/HD aikuisten psykologinen yksilökuntoutus - Psykologin käsikirja [Rehabilitation for adults with AD/HD - Manual]. Espoo, Finland: Rinnekoti-Säätiö [Rinnekoti Foundation]; 2009

25. Mirsky AF, Anthony BJ, Duncan CC, Ahearn MB, Kellam SG. Analysis of the elements of attention: a neuropsychological approach. Neuropsychol Rev. 1991;2(2):109-145.

26. Sohlberg MM, Mateer CA. Effectiveness of an attention training program. J Clin Exp Neuropsychol. 1987;9(2):117-130.

27. Sohlberg MM, Mateer CA. Improving attention and managing attentional problems: Adapting rehabilitation techniques to adults with ADHD. Ann NY Acad Sci. 2001;931:359-375.

28. Brown TE. Brown Attention-Deficit Disorder Scales for Adolescents and Adults. San Antonio, TX: The Psychological Corporation; 1996.
29. Kessler RC, Adler L, Ames M, et al. The World Health Organization Adult ADHD Self-Report Scale (ASRS): a short screening scale for use in the general population. Psychol Med. 2005;35(2):245-256.

30. Derogatis LR, Lipman RS, Covi L. SCL-90: An outpatient psychiatric rating scale - Preliminary report. Psychopharmacol Bull. 1973;9(1):13-28.

31. Beck AT, Steer RA, Brown GK. Beck Depression Inventory Second Edition (BDI II). San Antonio, TX.: The Psychological Corporation; 1996.

32. Endicott J, Nee J, Harrison W, Blumenthal R. Quality of Life Enjoyment and Satisfaction Questionnaire: a new measure. Psychopharmacol Bull. 1993;29(2):321-326.

33. Guy W. ECDEU Assessment Manual for Psychopharmacology. Rockville MD, US: Department of Health, Education, and Welfare, Public Health Service, Alcohol, Drug Abuse, and Mental Health Administration, NIMH Psychopharmacology Research Branch, Division of Extramural Research Programs; 1976.

34. Gualtieri CT, Johnson LG. Efficient allocation of attentional resources in patients with $\mathrm{AD} / \mathrm{HD}$ : maturational changes from age 10 to 29. J Atten Disord. 2006;9(3):534-542.

35. Gualtieri CT, Johnson LG. Reliability and validity of a computerized neurocognitive test battery, CNS Vital Signs. Arch Clin Neuropsychol. 2006;21(7):623-643.

36. Gualtieri CT, Johnson LG. A computerized test battery sensitive to mild and severe brain injury. Medscape J Med. 2008;10(4):90.
Neuropsychiatric Disease and Treatment

\section{Publish your work in this journal}

Neuropsychiatric Disease and Treatment is an international, peerreviewed journal of clinical therapeutics and pharmacology focusing on concise rapid reporting of clinical or pre-clinical studies on a range of neuropsychiatric and neurological disorders. This journal is indexed on PubMed Central, the 'PsycINFO' database and CAS, and is the official

\section{Dovepress}

journal of The International Neuropsychiatric Association (INA). The manuscript management system is completely online and includes a very quick and fair peer-review system, which is all easy to use. Visit http://www.dovepress.com/testimonials.php to read real quotes from published authors. 